\title{
Experimental investigation on thermocapillary drop migration at large Marangoni number in reduced gravity
}

\author{
Jing-Chang Xie *, Hai Lin, Pu Zhang, Fang Liu, Wen-Rui Hu \\ National Microgravity Lab/CAS, Institute of Mechanics, CAS, Beijing 100080, China
}

Received 27 April 2004; accepted 1 December 2004

Available online 21 February 2005

\begin{abstract}
Results from a space experiment on thermocapillary drop migration conducted on board the Chinese spacecraft ShenZhou-4 are presented in this paper. In the experiment, isolated drops of Fluorinert liquid moved in a matrix liquid of 5cst silicone oil at values of the Marangoni numbers (Ma) ranging up to 5500 and the interferometry images showed the temperature distribution inside the test cell. The drop migration velocity was measured. The experimental results show that the scaled drop migration velocity $V / V_{\text {YGB }}$ obviously decreases with Ma increasing the values up to 5500. The space experimental results are also compared with those from our early experiments, other space experiments, and some theoretical predictions.
\end{abstract}

(C) 2005 Elsevier Inc. All rights reserved.

Keywords: Interfacial tension; Thermocapillary migration; Microgravity

\section{Introduction}

A drop or a bubble will migrate in a matrix liquid subjected to a temperature gradient. The migration is caused by the nonuniform distribution of the interfacial tension on the interface due to the temperature gradient and is called thermocapillary migration or Marangoni migration. Young et al. first studied the thermocapillary migration of bubbles or drops with their linear analysis as the YGB model in the limitation when convective momentum and energy transport are both negligible $(\mathrm{Re} \rightarrow 0, \mathrm{Ma} \rightarrow 0)$ [1]. Since then, experiments on drop or bubble thermocapillary migration have been conducted under both normal-gravity and microgravity conditions [2-10]. Among these experiments, some of their results agree well with the linear prediction of Young et al. in the case of small drops migrating at small Reynolds numbers: for instance, the microgravity experiment on small drops $11 \pm 1.5 \mu \mathrm{m}$ in diameter have been performed on board a microgravity sounding rocket by Braun et al. [4]. The aver-

\footnotetext{
* Corresponding author.

E-mail address: jcxie@imech.ac.cn (J.-C. Xie).
}

age migration velocity $(0.22 \pm 0.03 \mu \mathrm{m} / \mathrm{s})$ well agreed with that given by the YGB model $(0.2 \pm 0.03 \mu \mathrm{m} / \mathrm{s})$. However, another experiment performed board the MASER sounding rocket by Wozniak obtained migration velocities for larger drops with diameters raging from 0.69 to $2.38 \mathrm{~mm}$ that are smaller than those given by the YGB linear prediction [5]. For this experiment, the Reynolds number is estimated to be larger than 1 according to the drop sizes and applied temperature gradient, and therefore the conditions for linear prediction are not satisfied. Afterward more attention was paid to drop Marangoni migration for larger Reynolds numbers or larger Marangoni numbers. For example, a ground-based experiment performed using a matched-density method by Xie et al. showed that the coupling migration velocities at moderate Marangoni numbers were not consistent with the one predicted by the linear model [6]. In the experiment, silicone oil and vegetable oil were used for the drop and continuous phases, respectively. Later on, using the same combination of drop and matrix liquid, they carried out the experiment of drop Marangoni migration at larger Marangoni numbers in the drop shaft facility in 1996 [8]. The maximum Marangoni number reached 1000 and much smaller migra- 
tion velocities, compared with the ones from YGB model, were observed in the experiment. The scaled velocity value $V / V_{\mathrm{YGB}}$, the ratio of the measured velocity to the one from the linear prediction, was found to decrease to around $20 \%$ as the Marangoni number increased to a value of 1000 . In addition, the space experiment of drop and bubble migration conducted by Balasubramaniam et al. on board the space shuttle during the IML-2 mission in 1994 [9] used Fluorinert liquid FC-75 and 50cst silicone oil as drop phase and continuous phase, respectively. In their experiment, the maximum Marangoni number at which the drop migrated is about 300 and the migration velocities are smaller than that from the linear prediction. The scaled velocity corresponding to the maximum Marangoni number in their experiment was decreased to approximately $25 \%$. Since the maximum Ma reached in their experiment is relatively small, Balasubramaniam and co-workers carried out another experiment on board the space shuttle in 1996. They used the same liquid as in the IML-2 mission for drops but a less viscous liquid, 10cst silicone oil, for the matrix phase and applied higher temperature gradients in order to increase the maximum Ma [10]. According to their experimental results, the scaled velocities of drop migration at Marangoni numbers less than about 400 were consistent with their early IML2 experiment. It can be seen from the data that the scaled velocity shows a decrease with increasing $\mathrm{Ma}$ in general and the data points distribute around a value of $30 \%$ as Ma closes to 400. But at higher value of $\mathrm{Ma}$ in the range of 1300-3700, the scaled velocity for each drop varies with the increase of Ma, showing an unsteady nature of migration process. Drop migration velocity is in a transient regime. From all the experiment results mentioned above, it can be seen that drop migration at large Marangoni numbers display a complex behavior, and when these experiment data are compared with the prediction from an asymptotic theory for $\mathrm{Re} \rightarrow \infty, \mathrm{Ma} \rightarrow \infty$ [11] and that from a numerical solution [12]; the latter suggests a sharp increase of the scaled velocity as the Marangoni number increases beyond about 90. Deviations can be noted for the scaled velocity at relatively large Marangoni numbers. The numerical results from an unsteady model are consistent with our early drop shaft experimental results, but only for the data with relatively small Reynolds numbers [13]. From the brief review above, it can be seen that the behavior of drop thermocapillary migration at large Marangoni number appears complex, and further studies are needed to observe the developing trend of drop migration velocity with increase of the values of Ma.

At the end of 2002, our experiment of drop migration was arranged and flown aboard China's spacecraft ShenZhou-4. The goal of the experiment is to expand the knowledge about the behaviors of drop migration at larger Marangoni numbers. Fluorinert liquid FC-75 and 5cst silicone oil were adopted as drop liquid and matrix phase, respectively, in this experiment. In this paper, the results from the space experiment are reported and the experimental data are compared with the results from other experiments, as well as some available theoretical predictions.

\section{Experimental apparatus and procedure}

To meet the requirements of the space experiment on board the Chinese unmanned spacecraft ShenZhou-4, we developed the apparatus, fixed in the recoverable capsule of the spacecraft, including a test cell, optical diagnostics and illumination system, video cameras, power supply, and computer control and servo systems. As shown in Fig. 1 as a schematic diagram, the main element of the apparatus

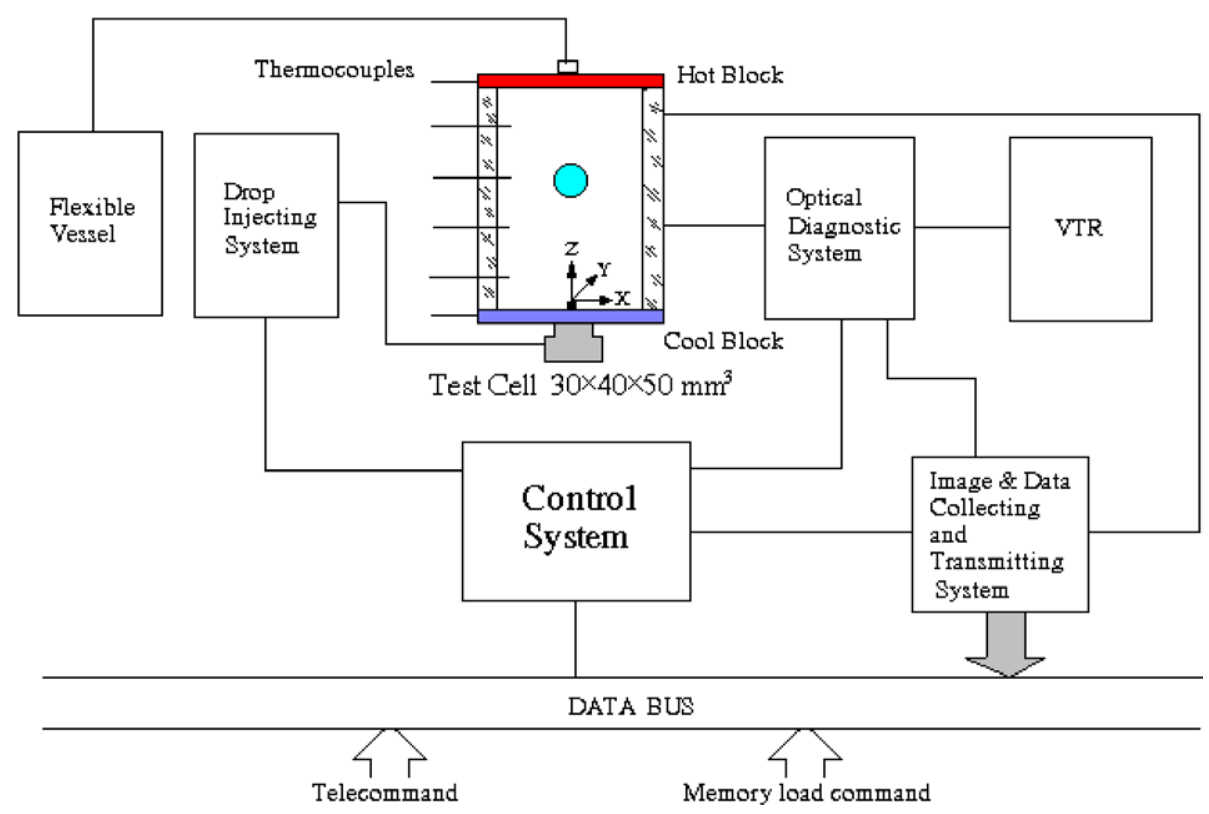

Fig. 1. Schematic diagram of the apparatus. 


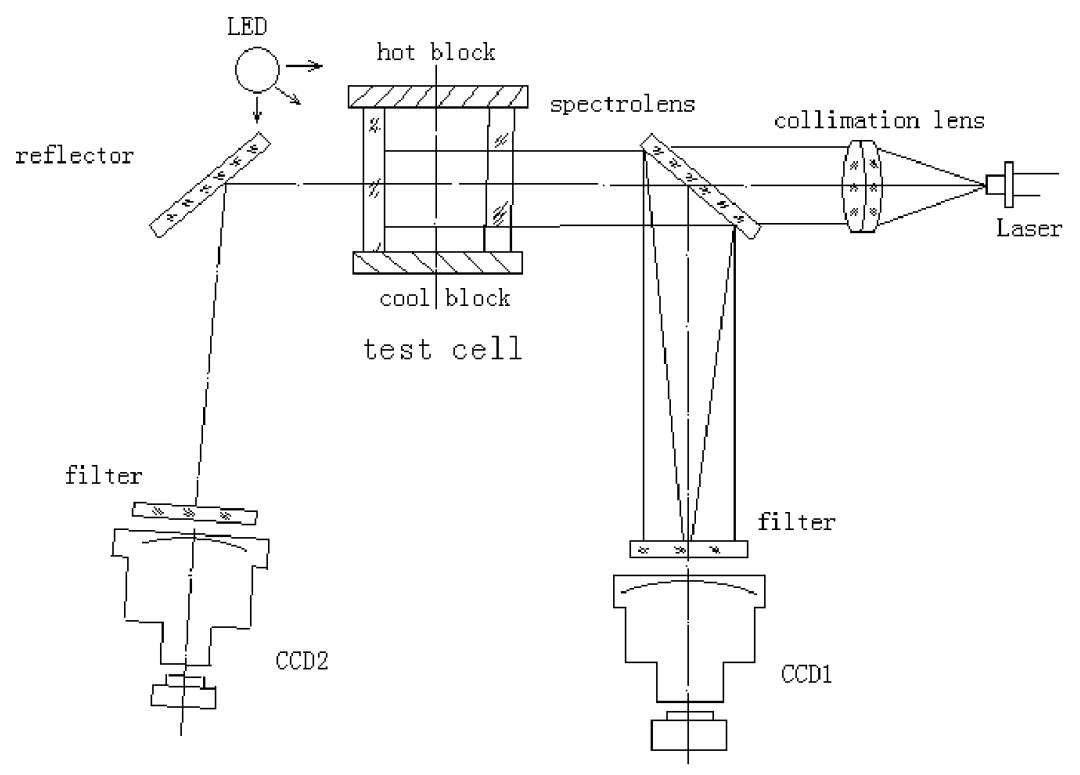

Fig. 2. Sketch of the optical system.

contains the test cell, drop injection system, optical diagnostic and illumination system, two CCD cameras, and the temperature measuring and controlling system. A computer, together with the electronic instruments, constitutes the controlling system, which has the function of carrying out the experiments according to the coded instructions, collecting and storing the scientific data, and communicating with the spacecraft. The recording device consists of two video tape recorders that record the moving drop trajectories and the interference images, respectively. The power supply device provides the power supply for all the facility.

At the heart of the apparatus there is a rectangular test cell with a cavity $50 \mathrm{~mm}$ in length and $40 \times 30 \mathrm{~mm}$ in cross section. Four 8-mm-thick glass walls forming the cavity are sealed with aluminum blocks at the top and bottom ends to enforce the temperature gradients along the vertical direction. A drop injector is fixed on the cold aluminum block, the main part of which is a specially designed dual-sleeve structure. The generator is connected via pipes with two syringes full of the drop liquid and the surrounding matrix liquid, respectively. The drop liquid is first fed through the inner pipe, forming a drop on the tip of it. There is a pause of $25 \mathrm{~s}$ after the injection of the drop and then the matrix liquid is injected through the annular gap between the inner and outer pipes to push the drop away from the tip of the injector. Two step motors drive the syringes, and the step motors are controlled by a computer servo system. Six thermocouples are used to measure the temperature. Two are fixed on the top and bottom aluminum blocks of the test cell, respectively, and another four thermocouples on one side glass wall.

The front and rear surfaces of the glass walls, combined with the lenses, the laser device $(\lambda=650 \mathrm{~nm})$, and the camera CCD 1, constitute a interferometer which is one part of optical diagnostic system. Two beams reflected from the front and rear surfaces of the test cell respectively form an in- terferential field. The beams reflected from the rear surface would pass through the liquid twice; therefore so-obtained interferential fringes carry the information of refractive index distribution related to the temperature field of the liquid. The interferometer can measure the variations of the temperature field in the fluid through the change of the interferential fringes. The sketch of the optical system is shown in Fig. 2. One of the advantages of the equi-thick interference system is its high stability, especially for the space experimental environment. Another CCD camera, CCD2, and the optical devices consist of the drop track camera system. Four light-emitting diodes are used for illuminating from the back.

In the process of the experiment, the volume of the matrix liquid in the test cell may expand when the liquid is heated to establish a temperature field. The drops injected into the matrix liquid would increase the total volume of the liquid as well. To offset the possible excessive liquid and keep the pressure steady in the test cell, a flexible vessel is used as a buffer reservoir that is connected with the test cell and sealed after the air in the vessel and the test cell is exhausted. The whole experiment process was basically controlled by the code embedded into the experimental unit on board unless intervened in by command instruction sent from the ground-based headquarters when extra operations were needed. The experimenter on the ground can make immediate or time-lapsed judgments according to the downlink information. Experimental data and telemetry data, such as the temperature data, the data displaying component status, and two channel images, were transmitted to the ground base during the experiment.

The heating process was activated $3 \mathrm{~h}$ before drop injection and then the temperature difference was controlled to get a linear temperature distribution along the vertical direction, the $z$-axis shown in the sketch, for each group of 
experiment run. For each group, four drops were injected at a time interval of several minutes between any two injections in order to reduce the disturbance of the temperature field caused by the predecessor's migration. All the traverses of drops were recorded by the two video cameras. According to the videotapes, the data were analyzed on the ground using an image processing system.

\section{Experimental results}

The experiment was designed for FC-75 Fluorinert drops migrating in a KF-96L series silicone oil of nominal viscosity 5 cst at two temperature gradients $\left(\left|\nabla T_{\infty}\right|\right)$ of 0.9 and $1.2 \mathrm{~K} / \mathrm{mm}$, respectively. Most of the physical parameters in the space experiment were measured. The dynamic viscosity, the heat conductivity, the density, and the coefficient of interfacial tension with temperature of the liquids at three typical temperatures are shown in Table 1 . The overall uncertainties are estimated to be within $10 \%$. The interfacial tension was measured over a temperature ranging from 20.9 to $68.8^{\circ} \mathrm{C}$ by a plate method using a Krüss $\mathrm{K} 12$ tensiometer. The results were fitted by straight line and the values of $\sigma_{\mathrm{T}}$ are $-0.044 \pm 0.002 \mathrm{dyn} /\left(\mathrm{cm}^{\circ} \mathrm{C}\right)$ for the $5 \mathrm{cst}$ silicone oil/Fluorinert FC-75 interface. The Reynolds number and Marangoni number are calculated by the definition

$\operatorname{Re}=\frac{V_{0} R}{v}$,

$M_{\mathrm{a}}=\frac{V_{0} R}{\kappa}$,

where $V_{0}=\left(-\sigma_{\mathrm{T}}^{\prime} R\left|\nabla T_{\infty}\right|\right) / \mu$ is the reference velocity, $R$ is the radius of the drop, $\sigma_{\mathrm{T}}^{\prime}$ is the temperature coefficient of interfacial tension, $\nabla T_{\infty}$ is the temperature gradient imposed on the liquid, and $\nu, \mu$, and $\kappa$ are kinematic viscosity, dynamic viscosity, and thermal diffusivity, respectively. The relevant information on the experiment is given in Table 2 .

In the experiment, the applied temperature gradient for each run was controlled and maintained. According to the readings of the thermocouples and the recorded video images from the interferometer, the temperature distribution along $z$-axis inside the test cell showed an approximate linear relationship with a less than $10 \%$ of the relative deviation. Near the hot wall of the test cell, larger temperature gradients appeared; see Fig. 3.

A total of 48 runs were completed on isolated drops, but only those velocity data of the drops whose migrations are judged not to be under the influence of the previous drop's motion (by disturbing the temperature field) are included in the velocity range in Table 2.

The typical sample plots of drop migration in two different temperature gradients are given in Fig. 4. Figs. $4 \mathrm{a}$ and $4 \mathrm{c}$ show the drop's position evolutions for two sets of drops of diameters 5.32, 2.85, and 3.23, $1.52 \mathrm{~mm}$ migrating along the $z$-axis direction of the test cell. The plots of corresponding velocities against time are provided in Figs. 4b, 4d. In these
Table 1

Property parameters at typical temperatures

\begin{tabular}{lllll}
\hline Fluid properties & & \multicolumn{3}{l}{ Temperature } \\
\cline { 3 - 5 } & & $25^{\circ} \mathrm{C}$ & $60^{\circ} \mathrm{C}$ & $80^{\circ} \mathrm{C}$ \\
\hline Silicone oil & $\mu\left(\mathrm{cp}=10^{-2} \mathrm{dyn} \mathrm{s} / \mathrm{cm}^{2}\right)$ & 4.268 & 2.491 & 1.869 \\
& $\lambda(\mathrm{mw} /(\mathrm{m} \mathrm{K}))$ & 111 & 106 & 104 \\
& $\rho\left(\mathrm{g} / \mathrm{cm}^{2}\right)$ & 0.910 & 0.878 & 0.859 \\
FC-75 & $\mu^{\prime}\left(\mathrm{cp}=10^{-2} \mathrm{dyn} \mathrm{s} / \mathrm{cm}^{2}\right)$ & 1.416 & 0.8517 & 0.6512 \\
& $\lambda^{\prime}(\mathrm{mw} /(\mathrm{m} \mathrm{K}))$ & 63 & 60.5 & 58.5 \\
FC-75/silicone oil & $\rho^{\prime}\left(\mathrm{g} / \mathrm{cm}^{2}\right)$ & 1.77 & 1.677 & 1.628 \\
& $\frac{\mathrm{d} \sigma}{\mathrm{d} T}(\mathrm{dyn} /(\mathrm{cm} \mathrm{K}))$ & $0.044 \pm 0.002$ & \\
\hline
\end{tabular}

Table 2

Parameter range of experimental data

\begin{tabular}{lllll}
\hline $\begin{array}{l}\text { Temperature } \\
\text { gradient } \\
(\mathrm{K} / \mathrm{mm})\end{array}$ & $\begin{array}{l}\text { Radius } \\
\text { range } \\
(\mathrm{mm})\end{array}$ & $\begin{array}{l}\text { Velocity } \\
\text { range } \\
V(\mathrm{~mm} / \mathrm{s})\end{array}$ & $\begin{array}{l}\text { Reynolds } \\
\text { number }\end{array}$ & $\begin{array}{l}\text { Marangoni } \\
\text { number }\end{array}$ \\
\hline 0.9 & $0.76-4.0$ & $1.28-3.80$ & $3.2-89.8$ & $148-4103$ \\
1.2 & $0.57-3.52$ & $1.14-7.44$ & $4.5-302.6$ & $145-5525$ \\
\hline
\end{tabular}

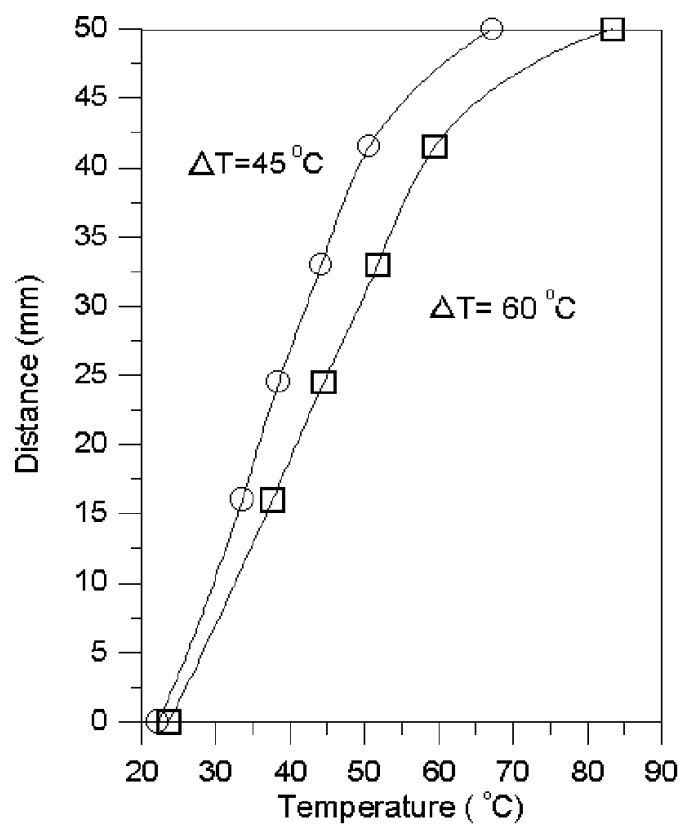

Fig. 3. Temperature distribution along $z$-axis inside the test cell.

drawings, $5 \%$ uncertainty estimated from the image processing system for the typical data points is included.

It can be seen from the curves that the drop migration was in an accelerating process all the time during the traverses with no exception found in all the experimental runs. Therefore, contrary to what was expected, there was no steady velocity reached and only instantaneous velocities could be measured in the experiments. We think one possible explanation for this finding is that the physical properties of the drop and matrix liquids varied in the experiments. Particularly, the viscosity of silicone oil as matrix liquid decreases with temperature and therefore results in the acceleration of a drop moving from a low temperature zone to a higher temperature 


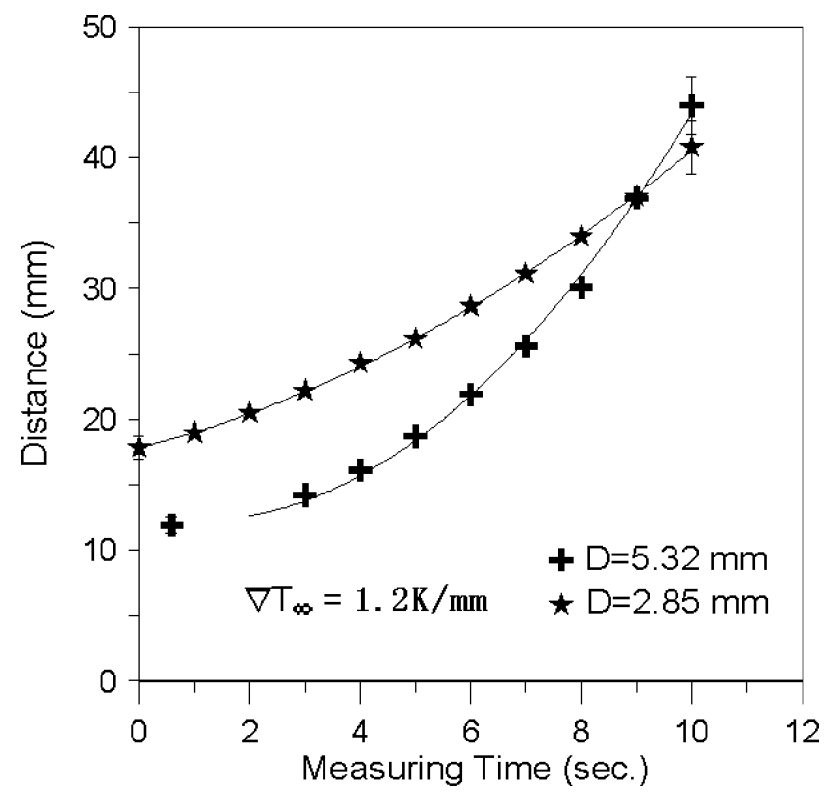

(a)

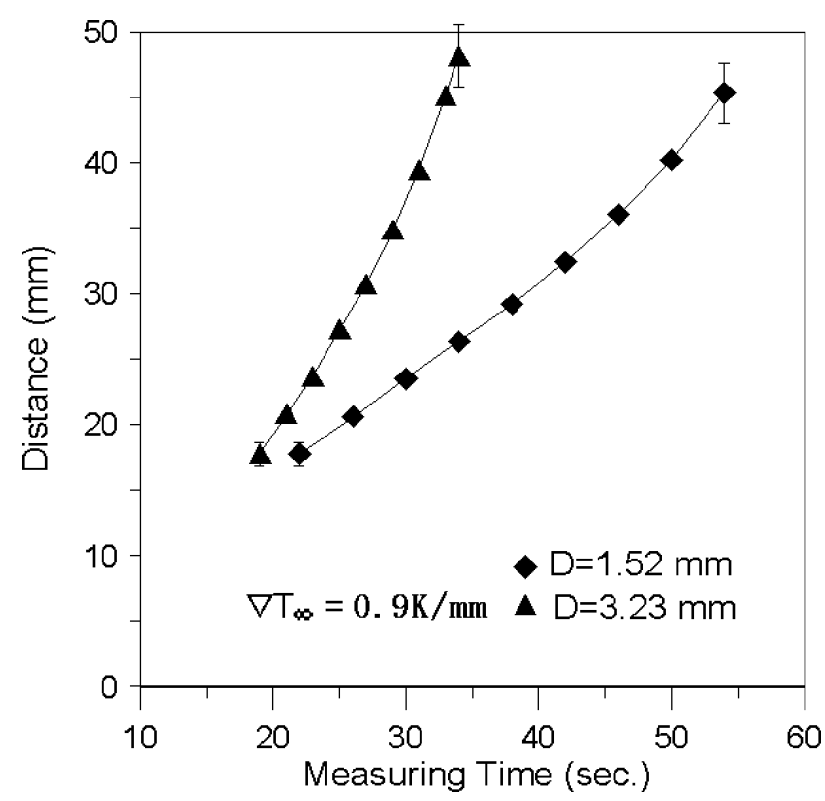

(c)

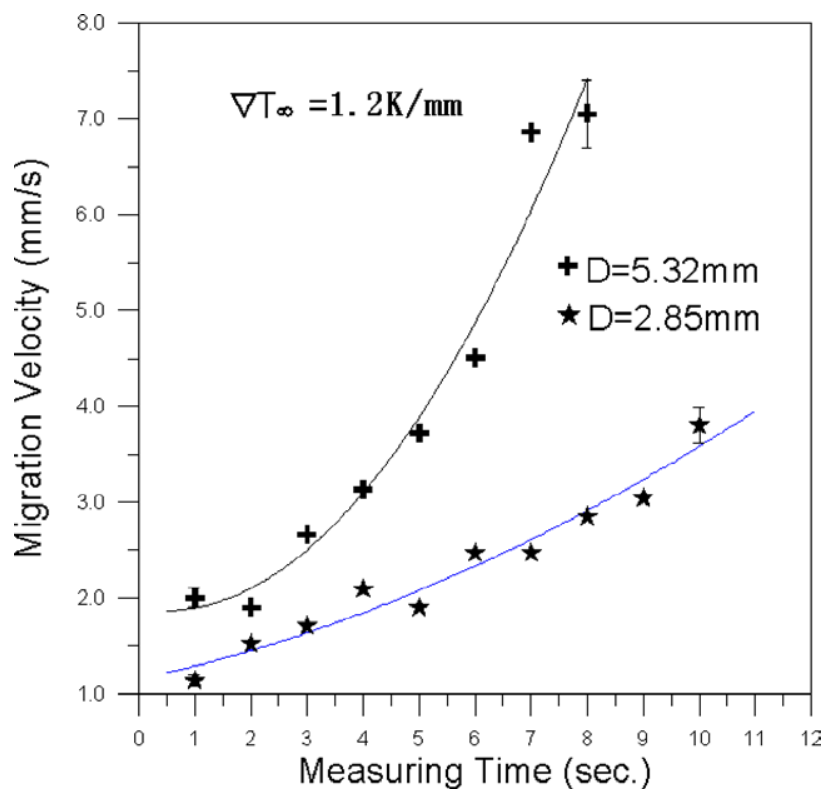

(b)

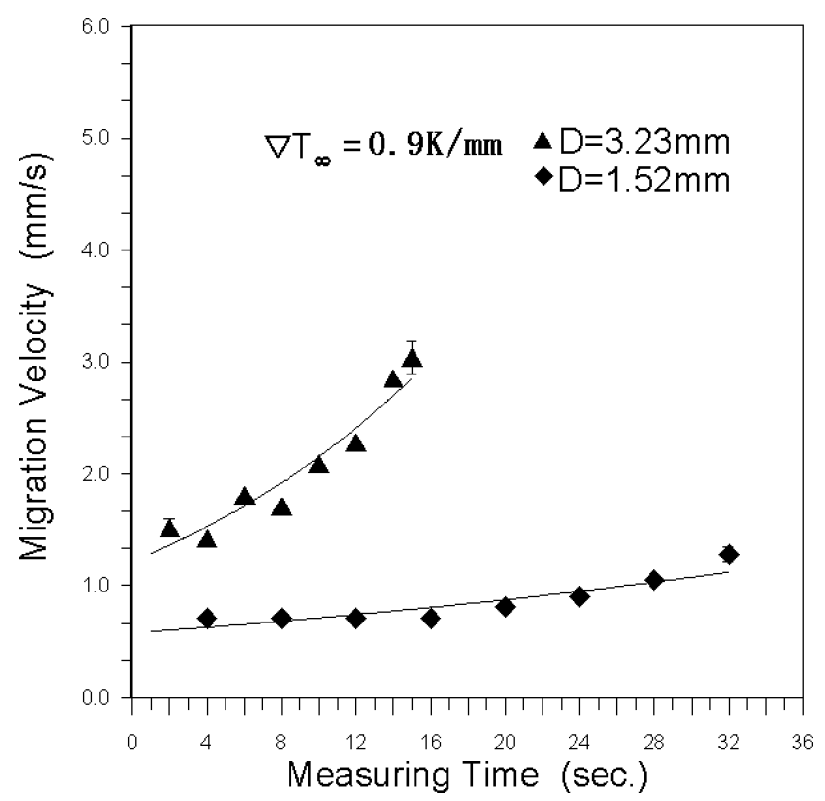

(d)

Fig. 4. Typical trajectories of two sets of drops plotted against time and the corresponding velocity profiles, respectively, in two temperature gradients $\left|\nabla T_{\infty}\right|=1.2 \mathrm{~K} / \mathrm{mm}$ (a) and (b) and $0.9 \mathrm{~K} / \mathrm{mm}$ (c) and (d).

one in the direction of the temperature gradient. According to Table 1 and the definition of reference velocity $V_{0}$, we can see that the value of $V_{0}$ at $25^{\circ} \mathrm{C}$ will become about 2.3 times larger than that at $80^{\circ} \mathrm{C}$ due to the change of viscosity of the matrix liquid. Besides, while a drop was moving at a relatively high velocity in a matrix liquid, the temperature inside the drop could not follow the temperature outside the drop during the traverse, even if both the heat transfer by conductivity and the inner convection could be relatively strong in these cases. There is insufficient time for the temperature gradient within the drop to achieve a steady distribution.

Since there were no steady velocities available in the space experiment, we present only the maximum migration velocities for all the data points for each drop. The data were taken for each drop at the location $8 \mathrm{~mm}$ from the warm end wall in order that the maximum velocities were measured within the linear temperature distribution region (see Fig. 3). In this way, the influences of both the end wall and the sharper temperature gradient near the warm wall on the drop migration velocity were minimized. The data on the scaled velocity, $V / V_{\mathrm{YGB}}$, plotted against the Marangoni number are shown in Fig. 5. Also plotted in the same drawing are the previous experimental data from our ground-based experiment and the drop shaft experiment to show the experimental results in an expanded range of Marangoni number. It should be mentioned that it is reasonable to use the local value of 


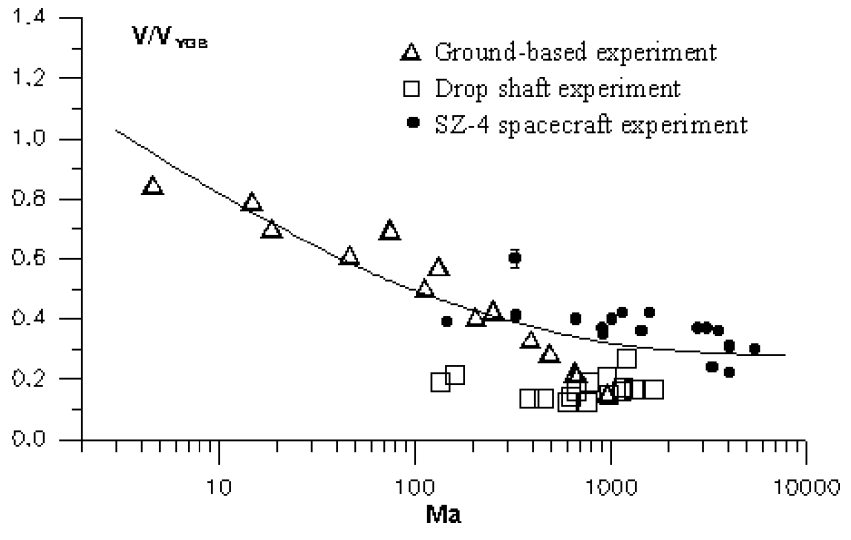

Fig. 5. Scaled velocity plotted against Marangoni number.

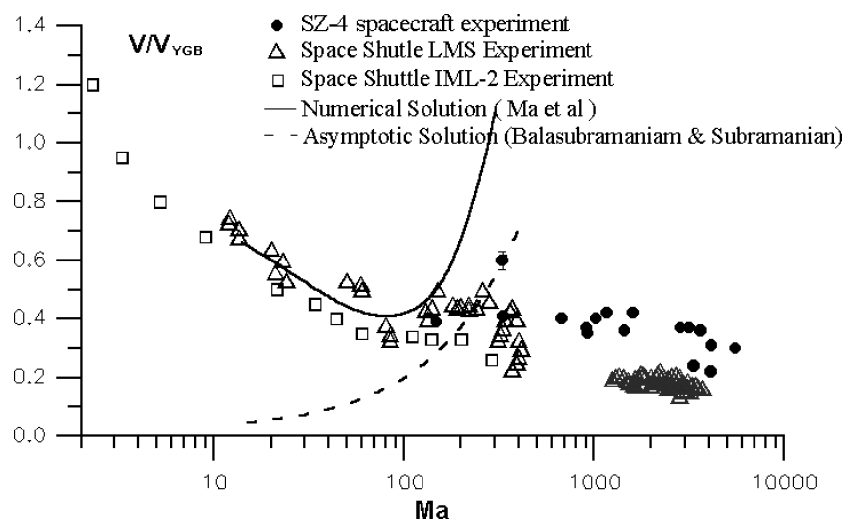

Fig. 6. Scaled velocity plotted against Marangoni number; data were obtained from the SZ-4 space experiment, IML-2 and LMS missions. Also plotted are predictions from an asymptotic theory and a numerical solution.

viscosity at the location where the maximum velocity of drop migration appeared to calculate $V_{\mathrm{YGB}}$ and the reference velocity $V_{0}$ in the Ma evaluation, in the case when the variation of viscosity causes the change of drop migration velocity. It can be seen from the experimental data, the maximum Marangoni number reached up to about 5500 and the curve in Fig. 5 shows a decreasing trend in general with increasing Ma and gradually approaches its asymptotic value: approximate $30 \%$.

For comparison purpose, Fig. 6 shows the data on scaled velocity obtained from three space experiments, including the data of Balasubramaniam et al. and Hadland et al. on the IML-2 and LMS missions. In all these experiments, the same liquid media. Fluorinert liquid FC-75 and silicone oil, are used as drop phase and continuous phase, respectively, but with different viscosities for silicone oil and temperature gradients applied to the liquid. Also given in Fig. 6 are a prediction from a asymptotic theory of Balasubramaniam and Subramanian [11] and a prediction from a numerical solution made by Ma [12]. When we put these space experimental data together, it can be seen that the curve still display a trend of decrease in general, even if data dispersion exists in the drawing. Besides, it is found that for large Marangoni numbers from 1300 to 3700 , in which the unsteady veloc- ity data obtained from LMS experiment are included [10], values of the scaled velocity from SZ-4 experiment results are obviously larger than those from LMS experiments. In this range the data from LMS experiments are given in a form of a data cluster for each drop due to that whose motion was in the transient regime. It can be also seen that at about $\mathrm{Ma} \approx 90$, the prediction of the numerical solution suggests a rapid increase of the scaled velocity with increasing Marangoni number. The asymptotic solution also predicts an increasing trend with increased of Ma. Apparently, there is a deviation of our data from the predictions: no distinct increase can be seen from the experimental data points even though the Marangoni number reaches up to about 5500. It should be noticed that because drop motion was accelerating during the traverses in the experiment, it is possible that the velocity would keep increasing to a higher value. In that case, the corresponding scaled velocities would likely be larger in the figure. But the present case is that no experiment has been performed in which sufficient velocity development of drop migration at large Marangoni numbers could be observed. It seems that it is also difficult to do such an experiment, considering various limitations to the experiments.

During the space experiment, all the corresponding interferometry images of drop migration were recorded in real time by using a VTR. However, to get more information from these images, further analysis are required. As a sample, Fig. 7 gives an original interferometry image showing the temperature field around a moving drop.

\section{Discussion}

The space experiment of drop thermocapillary migration presented here has expanded the range of Marangoni numbers. The experimental results show that the velocities of drop migration at moderate to lage Marangoni numbers up to $\mathrm{Ma}=5500$ are smaller than that given by the linear prediction of YGB model and the scaled velocities show, in general, a decreasing trend while Ma increases. Based on the present data obtained in the space experiments and the drop shaft experiments, there are obviously discrepancies between our experimental results and the predictions from the asymptotic theory and the numerical solution, not only in magnitude but even in trend. Because the drop migration was accelerated in the experiment reported here, only the maximum transient velocities can be determined experimentally in the space experiment. Apparently, although the maximum Marangoni number reaches about 5500 in this experiment, we still cannot infer from our present observation whether it is possible for the drop migration to reach steady velocities, and even if the steady velocities can be reached we still do not know whether they are consistent with the theoretical predictions mentioned above, in both cases of which the physical properties of the fluids are assumed constant. All these indicate that the drop thermocapillary migration at 


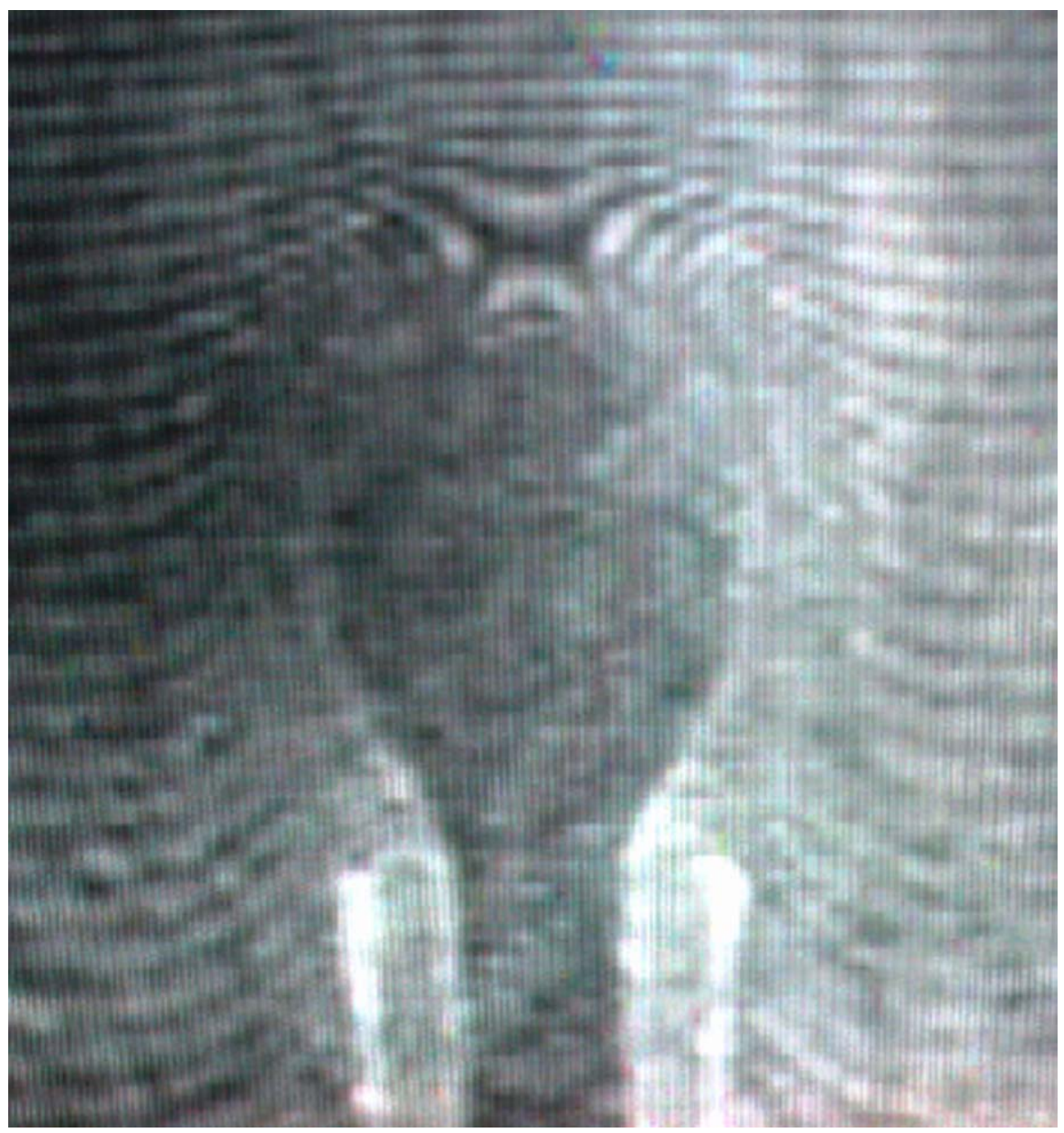

Fig. 7. Interferometry image of the temperature field around a moving drop.

large Marangoni numbers showed rather complex behavior and, further studies are still needed.

Finally, it shoud be taken into account that Fluorinert liquid FC-75 and silicone oil are miscible to some extent, especially at relatively high temperatures. The miscibility may result in the change of interfacial tension of the two liquids. At the beginning of the space experiment, the drops moving to the hot wall could be removed out of the test cell through a hole on the hot wall. But after several runs, it was found that some drops attached to the hot wall around the hole. The reason for this was that the drop's migration direction slightly deviated from a vertical straight line. In this case, those drops attached to the hot wall would be partially miscible with the matrix liquid. As a result the migration velocity of a drop moving into the miscible region would be affected correspondingly. We did see this phenomenon happening in the space experiment. We are going to discuss this issue in detail elsewhere. In addition, it was found that the drop migration direction very promptly and exactly followed the direction of the local temperature gradient during the traverse. As the disturbance of the temperature distribution caused by the traverse of the previous drop was not totally cleared up, the migration direction of the next drop would deviate from a straight trajectory.

\section{Acknowledgment}

The authors thank Professor Zhi-Wei Sun, NMLC, Institute of Mechanics, CAS, for helpful discussion.

\section{References}

[1] N.O. Young, J.S. Goldstein, M.J. Block, J. Fluid Mech. 11 (1959) 350.

[2] M. Hahnel, V. Delitzer, H. Eckelmann, Phys. Fluids A 1 (1989) 1460.

[3] M. Nallani, R.S. Subramanian, J. Colloid Interface Sci. 157 (1993) 24.

[4] B. Braun, C. Ikier, H. Klein, J. Colloid Interface Sci. 159 (1993) 515.

[5] G. Wozniak, J. Colloid Interface Sci. 141 (1991) 245.

[6] J.C. Xie, H. Lin, J.H. Han, W.R. Hu, Micrograv. Sci. Technol. 9 (2) (1996) 95.

[7] G. Wozniak, J. Siekmann, J. Srulijes, Z. Flugwiss. Weltraumforsch. 12 (1988) 137.

[8] J.C. Xie, H. Lin, J.H. Han, X.Q. Dong, W.R. Hu, A. Hirata, S.I. Nishizawa, M. Sakurai, Int. J. Heat Mass Transfer 41 (14) (1998) 2077.

[9] R. Balasubramaniam, C.E. Lacy, G. Wozniak, R.S. Subramanian, Phys. Fluid 8 (1996) 872.

[10] P.H. Hadland, R. Balasubramaniam, G. Wozniak, R.S. Subramanian, Exp. Fluids 26 (1999) 240.

[11] R. Balasubramaniam, R.S. Subramanian, Phys. Fluids 12 (4) (2000) 733.

[12] X. Ma, R. Balasubramaniam, R.S. Subramanian, Numer. Heat Transfer A 35 (1999) 291.

[13] G. Ronghui, H. Wenrui, J. Youlan, Acta Mech. Sin. 18 (3) (2002) 227. 